\title{
Exploring Data-driven Worked Examples for Block-based Programming
}

\author{
Rui Zhi \\ North Carolina State University \\ Raleigh, North Carolina \\ rzhi@ncsu.edu
}

\begin{abstract}
Empirical studies show that worked examples (WEs) are effective in improving students' learning efficiency in a variety of domains. I aim to create and evaluate data-driven intelligent WEs for novices using the Snap! block-based programming environment. First, I will design and evaluate WEs with self-explanation prompts in Snap! Then I will develop a data-driven method to generate WEs based on student solutions and compare it with manually-curated WEs.
\end{abstract}

\section{KEYWORDS}

block-based programming environment, data-driven, worked examples, self-explanation

\section{ACM Reference Format:}

Rui Zhi. 2018. Exploring Data-driven Worked Examples for Block-based Programming. In ICER '18: 2018 International Computing Education Research Conference, August 13-15, 2018, Espoo, Finland. ACM, New York, NY, USA, 2 pages. https://doi.org/10.1145/3230977.3231018

\section{PROGRAM CONTEXT}

I am a fourth year Ph.D. student in Computer Science at North Carolina State University. I will propose my dissertation in Fall 2018 and defend my dissertation in Fall 2019. I have led two studies investigating programming problems. In the first, I built and compared instructional designs for a programming game. In the second, I investigated the potential to algorithmically identify common features from student programs. Now I am developing the interface for worked examples (WEs) with self-explanation prompts in Snap! and will do a study to explore its effectiveness in this summer. I will refine the interface based on the study and evaluate this feature in a CS0 class with non-majors. I will also design algorithms to identify students' progress (i.e. to detect what parts of a problem a student has solved) and to generate data-driven WEs from student data.

\section{CONTEXT AND MOTIVATION}

My goal is to provide intelligent, data-driven support in blockedbased programming environments. My research is motivated by the effectiveness of worked examples (WEs) [6] and self explanations [5] in improving student learning in a variety of domains. However, WEs have not often been used for teaching programming, though

Permission to make digital or hard copies of part or all of this work for personal or classroom use is granted without fee provided that copies are not made or distributed for profit or commercial advantage and that copies bear this notice and the full citation on the first page. Copyrights for third-party components of this work must be honored.

For all other uses, contact the owner/author(s).

ICER '18, August 13-15, 2018, Espoo, Finland

(C) 2018 Copyright held by the owner/author(s)

ACM ISBN 978-1-4503-5628-2/18/08

https://doi.org/10.1145/3230977.3231018 many software engineers learn from and adapt examples to solve problems as they work. I plan to explore the effectiveness of datadriven WEs in novice block-based programming environments. A recent study shows that novices have difficulty in using examples to solve programming problems in a block-based programming environment [2]. I want to understand how to derive effective WEs from data, how students might use them, and whether the WEs help student learn programming. Based on the results of this work, I plan to augment Snap! with data-driven methods to detect student progress and provide relevant, timely interventions. Ideally, when a student gets stuck, the system I envision can provide corresponding example code to help them make progress, and the student can learn from the example by running the example code, reflecting on the output, explaining the code, and adapting it to their own solutions.

\section{BACKGROUND \& RELATED WORK}

Worked examples (WEs) are a common instructional support that helps novices learn more effectively than problem solving in various domains [6]. However, only a few studies explored how novices can effectively use WEs to learn block-based programming. In a pilot study for comparing different instructional designs in a programming game, we found that buggy WEs may be effective in helping students learn programming [7], but we had a small sample size. On the other hand, a study by Ichinco et al. shows that novices struggle to use WEs to solve block-based programming problems [2]. They found novices have difficulty in understanding the example and finding relevant blocks to finish the programming task. Along with WEs, self-explanation prompts help students foster greater understanding [5] and improve students' performance on problem solving [4]. Morrison et al. show that students who self-explained subgoal labels performed better than those who were not given subgoal labels [4]. Besides, the success of generating hints from student data motivates my research for leveraging the Hint Factory method [1] to generate WEs in block-based programming environments.

\section{STATEMENT OF THESIS/PROBLEM}

I will explore the effectiveness of WEs in helping novices learn block-based programming and develop a method to create datadriven WEs. My research questions are: RQ1: How effectively can students learn programming from WEs with self-explanation? RQ2: How can we generate data-driven WEs? RQ3: How do data-driven WEs compare with manually-curated WEs for student learning?

\section{RESEARCH GOALS \& METHODS}

I will do 3 studies to answer my research questions. In Study 1, I will develop an interface that allows students to view, run, and 
annotate WEs, and I evaluate it in a high school summer camp. The study uses a switching replications design. In this design, both groups will take a concept-based pre-test, solve a programming problem, take an isomorphic post-test, solve another programming problem, take a second isomorphic post-test, and solve a third programming problem. However, group A will be given a series of WEs during the first problem, and group B will receive WEs on the second problem. This means the first portion of the study serves as a controlled experiment, while the second portion ensures all students have access to the intervention and allows us to investigate the impact of WEs' ordering on students' problem solving. I will compare participants' problem-solving performance and learning gains between pre-test and the two post-tests. The third programming problem serves as a performance post-test to evaluate how well the WEs supported student programming skill development.

In Study 2, I will analyze student data from 2016-2018 to design methods for generating data-driven WEs - creating them from prior student data. I led a study to determine the feasibility of automatically classifying student programs according to features that they do and do not include [8]. In this study, 3 experts extracted features from student solutions, with each feature indicating a subset of correct code, and used those features to represent a student's code status. We also designed a data-driven way to classify student programs based on their program features. Our results show that the data-driven feature space overlaps with the expert feature space both qualitatively and quantitatively, and that students take similar strategies to accomplish programming assignments. These results suggest that it is feasible to detect a student's progress towards completing a problem, and to break apart sample student code to generate WEs for desired features in an open-ended problem. Based on this work, I will develop an algorithm to detect students' progress and integrate it into Snap!. Specifically, when a student is programming, the system can detect the features that are completed. When the student gets stuck, the system will select code from another prior student solution to present a WE that achieves a needed feature(s). I will design an algorithm to identify prior student work that achieves certain features, and use program traces to identify when a feature first appears in a solution. In this way, I can derive WEs that students can self-explain to determine how a new feature is added to existing code. I hypothesize that data-driven WEs can help improve students' problem-solving performance and learning gains. I will conduct a controlled experiment to test my hypothesis.

In Study 3, I will evaluate the data-driven WEs and compare it with manually-curated WEs in a CS0 class in Spring 2019. I will collect data and evaluate the manually-curated WEs from this class in Fall 2018. After finishing Study 2, I will integrate the algorithm into Snap! and generate data-driven WEs. Before each programming assignment, students will take a test to evaluate their learning. When they use the system to finish assignments, they will be randomly assigned to either the control group (with manually-curated WEs) or the experiment group (with data-driven WEs). I will compare their problem-solving performance, learning gains between preand post-tests, and final exam scores. I hypothesize that students receiving data-driven WEs will perform similarly to those receiving manually-curated WEs in the time it takes for them to write programs, and in learning gains. If successful, this will show that our system can use data to effectively select and present WEs.

\section{DISSERTATION STATUS}

I have gained an understanding of novices' programming behavior through a study to explore novices' debugging behavior in BOTS [3]. Our results show that students with higher scores on a self-explanation post-test made fewer code edits to fix a program. Recently, I led a study to explore the effect of WEs in BOTS [7]. In this study, we compared different instructional designs and our results suggest no difference of effectiveness on improving students' performance between WEs and instructional text, but participants using buggy WEs performed better. In addition to my previous work [8], the development of WEs with self-explanation prompts interface in Snap! is almost finished. I plan to propose my dissertation by the end of 2018 and finish my dissertation in December 2019. I hope the ICER doctoral consortium help me refine my proposal and research plan. In particular, I believe DC mentors will provide insight into how to best design the interface for WEs, and how to leverage WEs for novices in the context of solving a single problem and within the larger context of a semester-long programming course.

\section{EXPECTED CONTRIBUTIONS}

This work will provide insight into best practices for designing intelligent block-based programming environments. First, I will determine whether using WEs can help students learn programming concepts faster, and whether it helps them write programs to solve open-ended problems quicker. Second, I will investigate ways to derive relevant WEs from data, building on my work in using both expert and data-driven classification of the important aspects of student code solutions to open-ended problems. If successful, this will result in automatic detection of the features that students are working on and selection of prior student data that is similar to current student work. Third, I will compare the quality of data-driven WEs with that of manually-curated WEs in helping students learn programming.

\section{REFERENCES}

[1] Tiffany Barnes and John Stamper. 2010. Automatic hint generation for logic proof tutoring using historical data. Fournal of Educational Technology \& Society (2010), 3.

[2] Michelle Ichinco, Kyle J Harms, and Caitlin Kelleher. 2017. Towards Understanding Successful Novice Example User in Blocks-Based Programming. Fournal of Visual Languages and Sentient Systems (2017), 101-118.

[3] Zhongxiu Liu, Rui Zhi, Andrew Hicks, and Tiffany Barnes. 2017. Understanding problem solving behavior of 6-8 graders in a debugging game. Computer Science Education (2017), 1-29.

[4] Briana B Morrison, Lauren E Margulieux, and Mark Guzdial. 2015. Subgoals, context, and worked examples in learning computing problem solving. In ICER. 21-29.

[5] Marguerite Roy and Michelene TH Chi. 2005. The self-explanation principle in multimedia learning. The Cambridge handbook of multimedia learning (2005), 271-286.

[6] John Sweller. 2006. The worked example effect and human cognition. Learning and instruction (2006), 165-169.

[7] Rui Zhi, Nicholas Lytle, and Thomas W Price. 2018. Exploring Instructional Support Design in an Educational Game for K-12 Computing Education. In SIGCSE. 747-752.

[8] Rui Zhi, Thomas W. Price, Nicholas Lytle, Yihuan Dong, and Tiffany Barnes. (in press). Reducing the State Space of Programming Problems through Data-Driven Feature Detection. In EDM Workshop. 